\title{
Classifications and duality relations for several integral transforms
}

\author{
Xin Liang ${ }^{\mathrm{a}}$, Feng Gao ${ }^{\mathrm{a}, \mathrm{b}, *}$, Shan-Jie Su${ }^{\mathrm{a}}$, Zhen Wang ${ }^{\mathrm{c}}$, Xiao-Jun Yang ${ }^{\mathrm{a}, \mathrm{b}, \mathrm{c}}$ \\ a State Key Laboratory for Geomechanics and Deep Underground Engineering, China University of Mining and Technology, Xuzhou \\ 221116, China. \\ ${ }^{b}$ School of Mechanics and Civil Engineering, China University of Mining and Technology, Xuzhou 221116, China. \\ ${ }^{c}$ College of Mathematics and Systems Science, Shandong University of Science and Technology, Qingdao 266590, China.
}

Communicated by D. Baleanu

\begin{abstract}
In this paper, we classify several integral transforms into two categories according to the types of their kernel functions and two novel definitions of general integral transforms are suggested. Based on the general integral transforms, some of their basic properties are proved. In addition, the dualities between those two kinds of integral transforms are deducted and discussed in detail. The interesting coupling relations in symmetric form is illustrated graphically. The analysis shows that the classifications are reasonable and the dualities are significant. (C)2017 All rights reserved.
\end{abstract}

Keywords: Integral transforms, kernel functions, dualities.

2010 MSC: 44A99, 83C15, 76R50, 35Q99.

\section{Introduction}

Integral transforms (ITs) are known as the useful mathematical tools in solving the partial differential equations (PDEs) $[2,3,17,27]$. Specially, the diffusion equations and wave equations, as fundamental equations in applied mathematics and engineering, have been widely studied [7, 13, 24, 25]. According to the research shown in literatures $[6,9,16,21-23]$, several different ITs, such as Laplace transform (LT), Sumudu transform (ST), ELzaki transform (ET), Laplace-Carson transform (LCT), as well as two new integral transforms (NIT1 and NIT2), were equivalent in obtaining the analytic solutions of the diffusion or wave equations. However, because of the small differences in selection of the kernel functions, they differ in practical applications. For instance, Kang et al. [11] discovered that the using of LCT was more convenient to analyze the problem of American strangle options than LT and the numerical inversion of the LCT was more dominant than other ITs $[6,15]$. As well as, the ST has advantages in analyzing the non-constant coefficients wave equations $[7,13]$. In addition, LT and ST play the important roles in solving the fractional PDEs analytically or numerically $[1,10,14,26]$. Abdeljawad and Baleanu [1] discovered that LT may be applied to discuss the fractional derivatives with exponential kernel well and Kumar et al. [14]

\footnotetext{
*Corresponding author

Email addresses: xliang@cumt.edu.cn (Xin Liang), jsppw@sohu.com (Feng Gao), ssj_cumt@126.com (Shan-Jie Su), wangzhen_sd@126.com (Zhen Wang), dyangxiaojun@163.com (Xiao-Jun Yang)
}

doi:10.22436/jnsa.010.12.16 
showed us the great advantages of the local fractional ST methods in obtaining the numerical solutions of the Klein-Gordon equations. See [18, 19] for more extensive examples.

In fact, we find that these ITs are similar in form. It indicates that there may be a close connection between them. Presently, the research on the dualities of different ITs has been discussed, but it is mainly focused on the relations between LT and ST [4, 8, 12]. In particular, an interesting result discovered by Eltayeb and Kiliçman $[4,8,12]$ was that LT and ST interchange the image of $\sin (x)$ and $\cos (x)$. In addition, Liang et al. [16] graphically illustrated the duality relations of several ITs.

Recently, when we compare the kernel functions selected by these ITs, it seems that they can be classified. For example, LT, LCT, and NIT1 may belong to the same category and ST, ET, and NIT2 belong to the other same category. The details will be presented in the following sections. Meanwhile, based on the above ideas, a uniform, general or standardized definition is essential to integrate these ITs into the concise forms. In addition, what we have to mention is that the uniform, general or standardized ITs definitions may simplify and integrate the analysis of their basic properties and dualities.

In view of the above points, the purposes of this paper are to classify several similar ITs, give the general or standardized definitions, and explore their properties, especially the duality relations.

The structure of this paper is arranged as follows. Section 2 classifies several ITs into two types based on the commonalities and minor distinctions of their original definitions and proposes the more general or normalized definitions of these ITs. Meanwhile, some basic properties of the new general definitions are also discusses. Section 3 analyzes the duality relations between the two new general ITs in detail and emphasizes the functions of dualities. Section 4 summarizes the results.

\section{The classifications and general definitions of several integral transforms}

Note that the study in this paper is mainly focused on six similar ITs. For these ITs, the definition forms are similar, and the minor differences lie in the choice of the kernel functions. Before we classify these ITs, let us review their original definitions firstly.

\subsection{Original definitions of several integral transforms}

Definition 2.1. If $\phi(\lambda), \lambda>0$ is a real function, its NIT1 is described as follows [23]:

$$
\Omega(\xi)=N_{I}[\phi(\lambda)]=\frac{1}{\xi} \int_{0}^{\infty} \phi(\lambda) e^{-\xi \lambda} d \lambda, \xi>0,
$$

where $N_{I}$ is the NIT1 operator and $\xi^{-1} e^{-\xi \lambda}$ is the kernel function of NIT1.

Definition 2.2. If $\phi(\lambda), \lambda>0$ is a real function, its LT is defined as follows [5]:

$$
F(\xi)=L[\phi(\lambda)]=\int_{0}^{\infty} \phi(\lambda) e^{-\xi \lambda} \mathrm{d} \lambda, \xi>0,
$$

where $\mathrm{L}$ is the LT operator and $e^{-\xi \lambda}$ is the kernel function of LT.

Definition 2.3. If $\phi(\lambda), \lambda>0$ is a real function, its LCT is given as follows [6]:

$$
\mathrm{L}_{\mathrm{c}}(\xi)=\mathrm{C}[\phi(\lambda)]=\xi \int_{0}^{\infty} \phi(\lambda) \mathrm{e}^{-\xi \lambda} \mathrm{d} \lambda, \xi>0,
$$

where $\mathrm{C}$ is the LCT operator and $\xi e^{-\xi \lambda}$ is the kernel function of LCT.

Definition 2.4. If $\phi(\lambda), \lambda>0$ is a real function, its ST is given as follows [16]:

$$
\psi(\xi)=S[\phi(\lambda)]=\frac{1}{\xi} \int_{0}^{\infty} \phi(\lambda) e^{-\frac{\lambda}{\xi}} d \lambda, \xi>0,
$$

where $S$ is the LCT operator and $\xi^{-1} e^{-\frac{\lambda}{\xi}}$ is the kernel function of ST. 
Definition 2.5. If $\phi(\lambda), \lambda>0$ is a real function, its NIT2 is defined as follows [22]:

$$
\Phi(\xi)=\mathrm{Y}[\phi(\lambda)]=\int_{0}^{\infty} \phi(\lambda) e^{-\frac{\lambda}{\xi}} \mathrm{d} \lambda, \xi>0,
$$

where $Y$ is the NIT2 operator and $e^{-\frac{\lambda}{\xi}}$ is the kernel function of NIT2.

Definition 2.6. If $\phi(\lambda), \lambda>0$ is a real function, its ET is described as follows [9]:

$$
\mathrm{T}(\xi)=\mathrm{E}[\phi(\lambda)]=\xi \int_{0}^{\infty} \phi(\lambda) \mathrm{e}^{-\frac{\lambda}{\xi}} \mathrm{d} \lambda, \xi>0,
$$

where $\mathrm{E}$ is the ET operator and $\xi e^{-\frac{\lambda}{\xi}}$ is the kernel function of ET.

Table 1: The kernel functions of different ITs.

\begin{tabular}{|l|l|}
\hline Type 1 & Type 2 \\
\hline$\xi^{-1} e^{-\xi \lambda}$ & $\xi^{-1} e^{-\frac{\lambda}{\xi}}$ \\
$e^{-\xi \lambda}$ & $e^{-\frac{\lambda}{\xi}}$ \\
$\xi e^{-\xi \lambda}$ & $\xi e^{-\frac{\lambda}{\xi}}$ \\
\hline
\end{tabular}

According to the above definitions of different ITs, their kernel functions are summarized in Table 1. It is obvious that the kernel functions can be classified into two types clearly. In form, they are the products of power functions with different orders and exponential functions. Thus, we can describe them as $\xi^{m} e^{-\xi \lambda}$ and $\xi^{n} e^{-\lambda \xi^{-1}}$, where $m, n$ are the integers. Accordingly, two generalized definitions as general Laplace transform (GLT) and general Sumudu transform (GST) can be suggested as follows.

Definition 2.7.

$$
\mathrm{F}_{M}(\xi)=\mathrm{L}_{M}[\phi(\lambda)]=\int_{0}^{\infty} \phi(\lambda) \xi^{\mathrm{m}} e^{-\xi \lambda} \mathrm{d} \lambda, \xi>0,
$$

where $L_{M}$ is the GLT operator and $\xi^{m} e^{-\xi \lambda}$ is the kernel function of GLT.

Definition 2.8.

$$
\psi_{\mathrm{N}}(\xi)=\mathrm{S}_{\mathrm{N}}[\phi(\lambda)]=\int_{0}^{\infty} \phi(\lambda) \xi^{n} e^{-\frac{\lambda}{\xi}} \mathrm{d} \lambda, \xi>0,
$$

where $S_{N}$ is the GST operator and $\xi^{n} e^{-\frac{\lambda}{\xi}}$ is the kernel function of GST.

Clearly, when $m, n$ is $-1,0$ or 1 , the corresponding ITs are NIT1, LT, LCT, ST, NIT2 and ET, respectively. Thus, these ITs are integrated into two unified forms. Further, based on the above general definitions, we can analyze and discuss their properties systematically. In particular, they show an advantage in analyzing the dualities. The details will be presented in next section.

\subsection{Basic properties of GLT and GST}

In previous literatures $[6,9,16,21-23,25]$, the research on the properties of ITs was mainly focused on their linear property, differential property and convolution property, etc.. An important reason is that these properties play the key roles in solving differential equations. For example, in solving the PDEs, we usually transform them into the ordinary differential equations by using the ITs. In this process, the applications of linear property and differential property of ITs can simplify the solving process. In addition, Eltayeb and Kılıçman in literature [7], had emphasized the importance of convolution properties of ITs in solving the non-homogeneous wave with non-constant coefficients in detail. In this section, we will derive and prove these properties from the point of view of GLT and GST. Thus, the general form of these properties can be obtained. 
2.2.1. The properties of GLT

(T1) Linear property: If $F_{M 1}(\xi)=L_{M}\left[\phi_{1}(\lambda)\right], \xi>0, \lambda>0$ and $F_{M 2}(\xi)=L_{M}\left[\phi_{2}(\lambda)\right], \xi>0, \lambda>0$, then the linear property of GLT is given as:

$$
\mathrm{L}_{M}\left[\mathrm{a} \phi_{1}(\lambda)+\mathrm{b} \phi_{2}(\lambda)\right]=\mathrm{aF}_{\mathrm{M} 1}(\xi)+\mathrm{bF}_{\mathrm{M} 2}(\xi), \xi>0, \lambda>0,
$$

where $a$ and $b$ are the real constants.

(T2) Differential property: If $F_{M}(\xi)=L_{M}[\phi(\lambda)], \xi>0, \lambda>0$, the GLT of k-order derivatives of $\phi(\lambda)$ is given as:

$$
\begin{aligned}
\mathrm{L}_{M}\left[\phi^{(\mathrm{k})}(\lambda)\right]= & \xi^{\mathrm{k}} \mathrm{L}_{M}[\phi(\lambda)]-\xi^{\mathrm{m}+\mathrm{k}-1} \phi(0) \\
& -\xi^{\mathrm{m}+\mathrm{k}-2} \phi^{(1)}(0)-\cdots-\xi^{\mathrm{m}+1} \phi^{(\mathrm{k}-2)}(0)-\xi^{\mathrm{m}} \phi^{(k-1)}(0),
\end{aligned}
$$

where $\phi^{(k)}(\lambda)$ is k-order derivative of $\phi(\lambda)$.

(T3) Convolution property as defined in Definition 2.9.

Definition 2.9. Considering the real functions $\phi_{1}(\lambda), \lambda>0$, and $\phi_{2}(\lambda), \lambda>0$, their convolution is defined as $[7,16]$ :

$$
\phi_{1}(\lambda) * \phi_{2}(\lambda)=\int_{0}^{\lambda} \phi_{1}(\tau) \phi_{2}(\lambda-\tau) d \tau
$$

Thus, if $F_{M 1}(\xi)=L_{M}\left[\phi_{1}(\lambda)\right], \xi>0, \lambda>0$ and $F_{M 2}(\xi)=L_{M}\left[\phi_{2}(\lambda)\right], \xi>0, \lambda>0$, we have the GLT of the convolution as:

$$
\mathrm{L}_{M}\left[\phi_{1}(\lambda) * \phi_{2}(\lambda)\right]=\xi^{-m} \mathrm{~F}_{M 1}(\xi) \mathrm{F}_{M 2}(\xi) .
$$

Proof.

(T1)

$$
\begin{aligned}
\mathrm{L}_{M}\left[a \phi_{1}(\lambda)+b \phi_{2}(\lambda)\right] & =\int_{0}^{\infty}\left[a \phi_{1}(\lambda)+b \phi_{2}(\lambda)\right] \xi^{m} e^{-\xi \lambda} d \lambda \\
& =\int_{0}^{\infty} a \phi_{1}(\lambda) \xi^{m} e^{-\xi \lambda} d \lambda+\int_{0}^{\infty} b \phi_{2}(\lambda) \xi^{m} e^{-\xi \lambda} d \lambda=a F_{M 1}(\xi)+b F_{M 2}(\xi) .
\end{aligned}
$$

(T2)

$$
\begin{aligned}
\mathrm{L}_{M}\left[\phi^{(\mathrm{k})}(\lambda)\right]=\int_{0}^{\infty} \phi^{(\mathrm{k})}(\lambda) \xi^{\mathrm{m}} e^{-\xi \lambda} \mathrm{d} \lambda & =\left.\left[\phi^{(\mathrm{k}-1)}(\lambda) \xi^{\mathrm{m}} e^{-\xi \lambda}\right]\right|_{0} ^{\infty}+\xi \mathrm{L}_{M}\left[\phi^{(\mathrm{k}-1)}(\lambda)\right] \\
& =-\xi^{\mathrm{m}} \phi^{(\mathrm{k}-1)}(0)+\xi \mathrm{L}_{M}\left[\phi^{(\mathrm{k}-1)}(\lambda)\right] .
\end{aligned}
$$

Noting the recurrence relation between $L_{M}\left[\phi^{(k)}(\lambda)\right]$ and $L_{M}\left[\phi^{(k-1)}(\lambda)\right]$, we can obtain the following result:

$$
\begin{aligned}
\mathrm{L}_{M}\left[\phi^{(k)}(\lambda)\right]= & \xi^{\mathrm{k}} \mathrm{L}_{M}[\phi(\lambda)]-\xi^{\mathrm{m}+\mathrm{k}-1} \phi(0) \\
& -\xi^{\mathrm{m}+\mathrm{k}-2} \phi^{(1)}(0)-\cdots-\xi^{\mathrm{m}+1} \phi^{(\mathrm{k}-2)}(0)-\xi^{\mathrm{m}} \phi^{(\mathrm{k}-1)}(0) .
\end{aligned}
$$

(T3)

$$
\begin{aligned}
\mathrm{L}_{M}\left[\phi_{1}(\lambda) * \phi_{2}(\lambda)\right] & =\int_{0}^{\infty}\left[\phi_{1}(\lambda) * \phi_{2}(\lambda)\right] \xi^{\mathrm{m}} e^{-\xi \lambda} \mathrm{d} \lambda \\
& =\int_{0}^{\infty}\left[\int_{0}^{\lambda} \phi_{1}(\tau) \phi_{2}(\lambda-\tau) \mathrm{d} \tau\right] \xi^{\mathrm{m}} e^{-\xi \lambda} \mathrm{d} \lambda \\
& =\xi^{\mathrm{m}} \int_{0}^{\infty} \phi_{1}(\tau)\left[\int_{\tau}^{\infty} \phi_{2}(\lambda-\tau) e^{-\xi \lambda} \mathrm{d} \lambda\right] \mathrm{d} \tau
\end{aligned}
$$


Let $u=\lambda-\tau$. Then, we have

$$
\int_{\tau}^{\infty} \phi_{2}(\lambda-\tau) e^{-\xi \lambda} \mathrm{d} \lambda=\int_{0}^{\infty} \phi_{2}(u) e^{-\xi(u+\tau)} \mathrm{d} u=\xi^{-m} e^{-\xi \tau} F_{M 2}(\xi) .
$$

Substituting the Eq. (2.6) into Eq. (2.5), we can easily obtain that

$$
\mathrm{L}_{M}\left[\phi_{1}(\lambda) * \phi_{2}(\lambda)\right]=\xi^{-\mathrm{m}} \mathrm{F}_{\mathrm{M} 1}(\xi) \mathrm{F}_{M 2}(\xi) .
$$

\subsubsection{The properties of GST}

(T1) Linear property: If $\psi_{N 1}(\xi)=S_{N}\left[\phi_{1}(\lambda)\right], \xi>0, \lambda>0$ and $\psi_{N 2}(\xi)=S_{N}\left[\phi_{2}(\lambda)\right], \xi>0, \lambda>0$, then the linear property of GST is given as:

$$
S_{\mathrm{N}}\left[a \phi_{1}(\lambda)+b \phi_{2}(\lambda)\right]=a \psi_{N 1}(\xi)+b \psi_{N 2}(\xi), \xi>0, \lambda>0,
$$

where $a$ and $b$ are the real constants.

(T2) Differential property: If $\psi_{N}(\xi)=S_{N}[\phi(\lambda)], \xi>0, \lambda>0$, the GST of k-order derivatives of $\phi(\lambda)$ is given as:

$$
\begin{aligned}
S_{N}\left[\phi^{(k)}(\lambda)\right]= & \xi^{-k} S_{N}[\phi(\lambda)]-\xi^{n-(k-1)} \phi(0) \\
& -\xi^{n-(k-2)} \phi^{(1)}(0)-\cdots-\xi^{n-1} \phi^{(k-2)}(0)-\xi^{n} \phi^{(k-1)}(0),
\end{aligned}
$$

where $\phi^{(k)}(\lambda)$ is k-order derivative of $\phi(\lambda)$.

(T3) Convolution property: According to the definition of convolution in Eq. (2.4), if $\psi_{\mathrm{N} 1}(\xi)=S_{\mathrm{N}}\left[\phi_{1}(\lambda)\right]$, $\xi>0, \lambda>0$ and $\psi_{N 2}(\xi)=S_{N}\left[\phi_{2}(\lambda)\right], \xi>0, \lambda>0$, we have the GST of the convolution as:

$$
\psi_{\mathrm{N}}\left[\phi_{1}(\lambda) * \phi_{2}(\lambda)\right]=\xi^{-n} \psi_{\mathrm{N} 1}(\xi) \psi_{\mathrm{N} 2}(\xi) .
$$

Proof.

(T1)

$$
\begin{aligned}
\mathrm{S}_{\mathrm{N}}\left[a \phi_{1}(\lambda)+b \phi_{2}(\lambda)\right] & =\int_{0}^{\infty}\left[a \phi_{1}(\lambda)+b \phi_{2}(\lambda)\right] \xi^{n} e^{-\frac{\lambda}{\xi}} d \lambda \\
& =\int_{0}^{\infty} a \phi_{1}(\lambda) \xi^{n} e^{-\frac{\lambda}{\xi}} d \lambda+\int_{0}^{\infty} b \phi_{2}(\lambda) \xi^{n} e^{-\frac{\lambda}{\xi}} d \lambda=a \psi_{N 1}(\xi)+b \psi_{N 2}(\xi) .
\end{aligned}
$$

(T2)

$$
\begin{aligned}
S_{N}\left[\phi^{(k)}(\lambda)\right]=\int_{0}^{\infty} \phi^{(k)}(\lambda) \xi^{n} e^{-\frac{\lambda}{\xi}} \mathrm{d} \lambda & =\left.\left[\phi^{(k-1)}(\lambda) \xi^{n} e^{-\frac{\lambda}{\xi}}\right]\right|_{0} ^{\infty}+\xi^{-1} S_{N}\left[\phi^{(k-1)}(\lambda)\right] \\
& =-\xi^{n} \phi^{(k-1)}(0)+\xi^{-1} S_{N}\left[\phi^{(k-1)}(\lambda)\right] .
\end{aligned}
$$

Noting the recurrence relation between $S_{N}\left[\phi^{(k)}(\lambda)\right]$ and $S_{N}\left[\phi^{(k-1)}(\lambda)\right]$, we can easily obtain the result as follows

$$
\begin{aligned}
S_{N}\left[\phi^{(k)}(\lambda)\right]= & \xi^{-k} S_{N}[\phi(\lambda)]-\xi^{n-(k-1)} \phi(0) \\
& -\xi^{n-(k-2)} \phi^{(1)}(0)-\cdots-\xi^{n-1} \phi^{(k-2)}(0)-\xi^{n} \phi^{(k-1)}(0) .
\end{aligned}
$$

(T3)

$$
\begin{aligned}
\psi_{N}\left[\phi_{1}(\lambda) * \phi_{2}(\lambda)\right] & =\int_{0}^{\infty}\left[\phi_{1}(\lambda) * \phi_{2}(\lambda)\right] \xi^{n} e^{-\frac{\lambda}{\xi}} d \lambda \\
& =\int_{0}^{\infty}\left[\int_{0}^{\lambda} \phi_{1}(\tau) \phi_{2}(\lambda-\tau) d \tau\right] \xi^{n} e^{-\frac{\lambda}{\xi}} d \lambda \\
& =\xi^{n} \int_{0}^{\infty} \phi_{1}(\tau)\left[\int_{\tau}^{\infty} \phi_{2}(\lambda-\tau) e^{-\frac{\lambda}{\xi}} d \lambda\right] d \tau
\end{aligned}
$$


Let $u=\lambda-\tau$. Then, we have

$$
\int_{\tau}^{\infty} \phi_{2}(\lambda-\tau) e^{-\frac{\lambda}{\xi}} \mathrm{d} \lambda=\int_{0}^{\infty} \phi_{2}(u) e^{-\frac{u+\tau}{\xi}} \mathrm{d} u=\xi^{-n} e^{-\frac{\tau}{\xi}} \psi_{\mathrm{N} 2}(\xi)
$$

Substituting the Eq. (2.9) into Eq. (2.8), we can easily obtain that

$$
\psi_{\mathrm{N}}\left[\phi_{1}(\lambda) * \phi_{2}(\lambda)\right]=\xi^{-n} \psi_{\mathrm{N} 1}(\xi) \psi_{\mathrm{N} 2}(\xi) .
$$

Clearly, when the values of $m$ or $n$ are given, we can conveniently obtain the basic properties for a particular IT. For example, when $m=-1$ or $n=1$, correspondingly, the NIT1 or ET of one-order differential of the function $\phi(\lambda)(k=1$ for Eq. (2.3) and Eq. (2.7)) can be obtained as:

$$
\mathrm{L}_{M}\left[\phi^{\prime}(\lambda)\right]=\xi \mathrm{L}_{M}[\phi(\lambda)]-\frac{\phi(0)}{\xi}
$$

and

$$
S_{N}\left[\phi^{\prime}(\lambda)\right]=\frac{S_{N}[\phi(\lambda)]}{\xi}-\xi \phi(0)
$$

Eqs. (2.10) and (2.11), by contrast, are same to the result presented in [9, 16]. Similarly, it remains easy to obtain other properties mentioned above for other ITs, when $m$ and $n$ take other values. Therefore, the GLT and GST are significant to integrate the ITs. Meanwhile, they are also convenient to study the properties of ITs.

\section{Duality relations between GLT and GST}

\subsection{Proof and analysis of the duality relations}

As presented in many literatures $[4,8,12,16,20]$, there is a deep connection between different ITs. However, rigorous proof of the dualities is lacking due to the forms of ITs are various. The classification and general definitions for different ITs in this paper seem to provide the effective means to study the dualities. In this section, we will prove the dualities and obtain an interesting conclusion.

From the definitions of GLT and GST, we can easily have:

$$
F_{M}(\xi)=L_{M}[\phi(\lambda)]=\int_{0}^{\infty} \phi(\lambda) \xi^{m} e^{-\xi \lambda} d \lambda=\xi^{m+n} \int_{0}^{\infty} \phi(\lambda)\left(\xi^{-1}\right)^{n} e^{-\frac{\lambda}{\xi^{-1}}} d \lambda=\xi^{m+n} \psi_{N}\left(\xi^{-1}\right)
$$

If we make $\xi$ take its negative first power $\xi^{-1}$, Eq. (3.1) becomes that

$$
\mathrm{F}_{M}\left(\xi^{-1}\right)=\xi^{-(\mathrm{m}+\mathrm{n})} \psi_{\mathrm{N}}(\xi)
$$

or

$$
\psi_{N}(\xi)=\xi^{m+n} F_{M}\left(\xi^{-1}\right)
$$

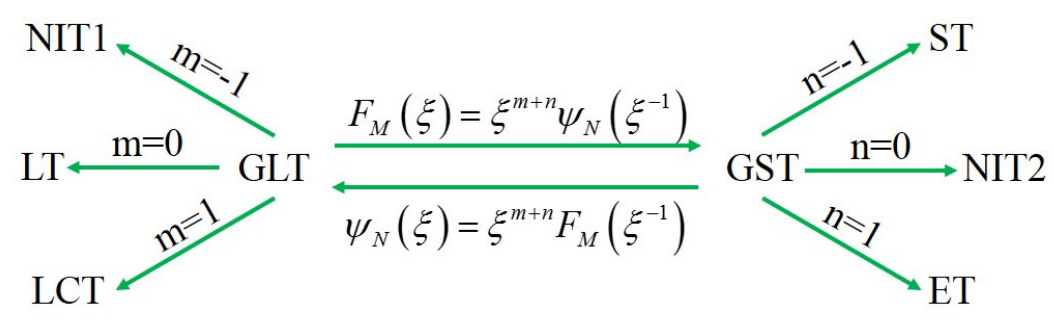

Figure 1: The dualities graph between GLT and GST. 
Contrasting Eqs. (3.1) and (3.2), we discover that the forms of the dualities between GLT and GST are completely symmetric. Correspondingly, we illustrate them in Fig. 1. Thus, if we make $m$ and $n$ take different values, the dualities between different ITs can be obtained. For example, if $m=0, n=-1$ or $\mathrm{m}=-1, \mathrm{n}=-1$, the dualities between LT and ST, as well as NIT1 and ST, can be shown as:

$$
F(\xi)=\xi^{-1} \psi\left(\xi^{-1}\right)
$$

and

$$
\Omega(\xi)=\xi^{-2} \psi\left(\xi^{-1}\right) .
$$

The results are in agreement with previous reports in [16]. Similarly, it remains easy to obtain the dualities for other ITs when $m$ and $n$ take other values. Note that we do not discuss the dualities of ITs belonging only to GLT or GST here. One reason is that there is no complete symmetry for their dualities, and on the other hand, there is only a power coefficient difference in their coupling relations. For a clearer understanding, ITs of the trigonometric function mentioned in $[8,13]$ provide the intuitional example. Specifically, according to the illustrations by Eltayeb and Kiliçman [8], the strong duality relations between LT and ST of $\sin (\lambda)$ and $\cos (\lambda)$ can be presented as:

$$
\mathrm{L}[\sin (\lambda)]=\mathrm{S}[\cos (\lambda)]
$$

and

$$
\mathrm{L}[\cos (\lambda)]=\mathrm{S}[\sin (\lambda)] .
$$

Inspired by this, here, in view of Eqs. (2.1) and (2.2), we derive GLT and GST of $\sin (\lambda)$ and $\cos (\lambda)$ as:

$$
\begin{aligned}
& L_{M}[\sin (\lambda)]=L_{M}\left[\frac{1}{2 j}\left(e^{j \lambda}-e^{-j \lambda}\right)\right]=\frac{\xi^{m}}{2 j}\left[\frac{1}{\xi-j}-\frac{1}{\xi+j}\right]=\frac{\xi^{m}}{\xi^{2}+1^{\prime}}, \\
& L_{M}[\cos (\lambda)]=L_{M}\left[\frac{1}{2}\left(e^{j \lambda}+e^{-j \lambda}\right)\right]=\frac{\xi^{m}}{2}\left[\frac{1}{\xi-j}+\frac{1}{\xi+j}\right]=\frac{\xi^{m+1}}{\xi^{2}+1^{\prime}} \\
& \psi_{N}[\sin (\lambda)]=S_{N}\left[\frac{1}{2 j}\left(e^{j \lambda}-e^{-j \lambda}\right)\right]=\frac{\xi^{n+1}}{2 j}\left[\frac{1}{1-j \xi}-\frac{1}{1+j \xi}\right]=\frac{\xi^{n+2}}{1+\xi^{2}}, \\
& \psi_{N}[\cos (\lambda)]=S_{N}\left[\frac{1}{2}\left(e^{j \lambda}+e^{-j \lambda}\right)\right]=\frac{\xi^{n+1}}{2}\left[\frac{1}{1-j \xi}+\frac{1}{1+j \xi}\right]=\frac{\xi^{n+1}}{1+\xi^{2}} .
\end{aligned}
$$

Then, if $L_{M}[\sin (\lambda)]=\psi_{N}[\cos (\lambda)]$ and $L_{M}[\cos (\lambda)]=\psi_{N}[\sin (\lambda)]$, we can obtain an interesting result as:

$$
\left\{\begin{array}{l}
\mathrm{m}=\mathrm{n}+1, \\
\mathrm{~m}+1=\mathrm{n}+2,
\end{array} \Rightarrow \mathrm{m}-\mathrm{n}=1\right.
$$

However, for the only GLT or GST, when $m$ or $n$ take different values, we cannot achieve the above symmetry relations. For example, when $m=1$ and 0 , we have

$$
C[\sin (\lambda)]=L[\cos (\lambda)],
$$

but,

$$
C[\cos (\lambda)] \neq L[\sin (\lambda)] .
$$

Therefore, it also indicates that the classification in this paper is reasonable. In addition, noticing the result of Eq. (3.3), we discover that the LCT $(m=1)$ and ET $(n=0)$ have also the symmetry coupling relations.

\subsection{The functions of duality relations}

The symmetry of duality relations between GLT and GST indicates that these two kinds of ITs may transform each other. In practice, for different problems, it is important to choose the proper ITs. When we know the property of a kind of ITs, the dualities may serve as the convenient means to obtain the property of another ITs [4]. In order to verify the feasibility of the above ideas, based on the differential 
properties of GLT, an example of obtaining the differential properties of GST is presented below.

Firstly, let

$$
\begin{aligned}
\mathrm{L}_{M}\left[\phi^{(\mathrm{k})}(\lambda)\right] & =\mathrm{F}_{M K}(\xi), \mathrm{L}_{M}[\phi(\lambda)]=\mathrm{F}_{M}(\xi) \\
\mathrm{S}_{\mathrm{N}}\left[\phi^{(\mathrm{k})}(\lambda)\right] & =\psi_{N K}(\xi), \mathrm{S}_{\mathrm{N}}[\phi(\lambda)]=\psi_{\mathrm{N}}(\xi)
\end{aligned}
$$

and

$$
\begin{aligned}
\mathrm{F}_{M K}(\xi) & =\xi^{\mathrm{m}+\mathrm{n}} \psi_{N K}\left(\xi^{-1}\right), & \psi_{N K}(\xi) & =\xi^{\mathrm{m}+\mathrm{n}} \mathrm{F}_{M K}\left(\xi^{-1}\right), \\
\mathrm{F}_{M}(\xi) & =\xi^{\mathrm{m}+\mathrm{n}} \psi_{\mathrm{N}}\left(\xi^{-1}\right), & \psi_{\mathrm{N}}(\xi) & =\xi^{\mathrm{m}+\mathrm{n}} \mathrm{F}_{M}\left(\xi^{-1}\right) .
\end{aligned}
$$

Making $\xi$ take its negative first power $\xi^{-1}$ and substituting Eq. (2.3) into Eqs. (3.4) and (3.5), we have

$$
\begin{aligned}
S_{N}\left[\phi^{(k)}(\lambda)\right]= & \psi_{N K}(\xi) \\
= & \xi^{m+n}\left[\begin{array}{l}
\xi^{-k} F_{M}\left(\xi^{-1}\right)-\xi^{-m-(k-1)} \phi(0) \\
-\xi^{-m-(k-2)} \phi^{(1)}(0)-\cdots-\xi^{-m-1} \phi^{(k-2)}(0)-\xi^{-m} \phi^{(k-1)}(0)
\end{array}\right] \\
= & \xi^{m+n}\left[\begin{array}{l}
\xi^{-k} \xi^{-(m+n)} \psi_{N}(\xi)-\xi^{-m-(k-1)} \phi(0) \\
-\xi^{-m-(k-2)} \phi^{(1)}(0)-\cdots-\xi^{-m-1} \phi^{(k-2)}(0)-\xi^{-m} \phi^{(k-1)}(0)
\end{array}\right] \\
= & \xi^{-k} S_{N}[\phi(\lambda)]-\xi^{n-(k-1)} \phi(0) \\
& -\xi^{n-(k-2)} \phi^{(1)}(0)-\cdots-\xi^{n-1} \phi^{(k-2)}(0)-\xi^{n} \phi^{(k-1)}(0) .
\end{aligned}
$$

Eq. (3.6), by contrast, is same to Eq. (2.7). On the contrary, we can also obtain the differential properties of GLT from GST by using their dualities. Obviously, the applications of dualities simplify the proof process. Certainly, other properties of ITs can be derived via the dualities. Therefore, the research on dualities is important.

\section{Conclusions}

In this paper, several ITs based on the similarity and distinction of their kernel functions are classified into two categories. Meanwhile, these kernel functions were integrated and two general ITs definitions, called GLT and GST, were suggested. It was convenient to uniformly or systematically study the basic properties of different ITs by applying GLT and GST. In addition, the dualities between GLT and GST are prove and the interesting symmetric coupling forms are illustrated graphically. The example illustrating the relations between GLT and GST of trigonometric functions indicates that there are only the symmetric dualities for these two kinds of ITs, but there are not any for ITs belonging only to GLT or GST. Thus, the symmetric dualities existing only between these two kinds of ITs also reflected the validity of classification in this article. Finally, the dualities are proved to be important and convenient to convert or derive the properties of GLT and GST each other.

\section{Acknowledgment}

This research was financially supported by the Fundamental Research Funds for the Central Universities (No. 2017BSCXA21) and the Postgraduate Research \& Practice Innovation Program of Jiangsu Province (No. KYCX17_1531).

\section{References}

[1] T. Abdeljawad, D. Baleanu, On fractional derivatives with exponential kernel and their discrete versions, Rep. Math. Phys., 80 (2017), 11-27. 1

[2] A. Atangana, Extension of the Sumudu homotopy perturbation method to an attractor for one-dimensional Keller-Segel equations, Appl. Math. Model., 39 (2015), 2909-2916. 1

[3] A. Atangana, J. F. Gómez-Aguilar, A new derivative with normal distribution kernel: theory, methods and applications, Phys. A, 476 (2017), 1-14. 1 
[4] F. B. M. Belgacem, A. A. Karaballi, S. L. Kalla, Analytical investigations of the Sumudu transform and applications to integral production equations, Math. Probl. Eng., 2003 (2003), 103-118. 1, 3.1, 3.2

[5] G. Doetsch, L. Debnath, Introduction to the theory and application of the Laplace transformation, Translated from the second German edition by Walter Nader, Springer-Verlag, New York-Heidelberg, (1974). 2.2

[6] C. Donolato, Analytical and numerical inversion of the Laplace-Carson transform by a differential method, Comput. Phys. Comm., 145 (2002), 298-309. 1, 2.3, 2.2

[7] H. Eltayeb, A. K1lıçman, A note on solutions of wave, Laplace's and heat equations with convolution terms by using a double Laplace transform, Appl. Math. Lett., 21 (2008), 1324-1329. 1, 2.2, 2.9

[8] H. Eltayeb, A. Kıliçman, On double Sumudu transform and double Laplace transform, Malays. J. Math. Sci., 4 (2010), 17-30. 1, 3.1, 3.1

[9] T. M. Elzaki, Application of new transform Elzaki transform to partial differential equations, Global J. Pure Appl. Math., 7 (2011), 65-70. 1, 2.6, 2.2, 2.2.2

[10] F. Jarad, E. Uğurlu, T. Abdeljawad, D. Baleanu, On a new class of fractional operators, Adv. Difference Equ., 2017 (2017), 16 pages. 1

[11] M.-J. Kang, J.-K. Jeon, H.-J. Han, S.-M. Lee, Analytic solution for American strangle options using Laplace-Carson transforms, Commun. Nonlinear Sci. Numer. Simul., 47 (2017), 292-307. 1

[12] A. Kılıçman, H. Eltayeb, K. A. M. Atan, A note on the comparison between Laplace and Sumudu transforms, Bull. Iranian Math. Soc., 37 (2011), 131-141. 1, 3.1

[13] A. Kılicman, H. E. Gadain, On the applications of Laplace and Sumudu transforms, J. Franklin Inst., 347 (2010), 848 862. $1,3.1$

[14] D. Kumar, J. Singh, D. Baleanu, A hybrid computational approach for Klein-Gordon equations on Cantor sets, Nonlinear Dynam., 87 (2017), 511-517. 1

[15] M. Lévesque, M. D. Gilchrist, N. Bouleau, K. Derrien, D. Baptiste, Numerical inversion of the Laplace-Carson transform applied to homogenization of randomly reinforced linear viscoelastic media, Comput. Mech., 40 (2008), 771-789. 1

[16] X. Liang, F. Gao, Y.-N. Gao, X.-J. Yang, Applications of a novel integral transform to partial differential equations, J. Nonlinear Sci. Appl. , 10 (2017), 528-534. 1, 2.4, 2.2, 2.9, 2.2.2, 3.1, 3.1

[17] X. Liang, G. N. Liu, S. J. Su, Applications of a novel integral transform to the convection-dispersion equations, Therm. Sci., 21 (2017), S233-S240. 1

[18] J. Singh, D. Kumar, M. A. Qurashi, D. Baleanu, A novel numerical approach for a nonlinear fractional dynamical model of interpersonal and romantic relationships, Entropy, 19 (2017), 17 pages. 1

[19] J. Singh, R. Swroop, D. Kumar, A computational approach for fractional convection-diffusion equation via integral transforms, Ain Shams Eng. J., (2016), (in press). 1

[20] H. M. Srivastava, M.-J. Luo, R. K. Raina, A new integral transform and its applications, Acta Math. Sci. Ser. B Engl. Ed., 35 (2015), 1386-1400. 3.1

[21] S. Weerakoon, Application of Sumudu transform to partial differential equations, Internat. J. Math. Ed. Sci. Tech., 25 (1994), 277-283. 1, 2.2

[22] X.-J. Yang, A new integral transform method for solving steady heat-transfer problem, Therm. Sci., 20 (2016), S639-S642. 2.5

[23] X.-J. Yang, A new integral transform with an application in heat-transfer problem, Therm. Sci., 20 (2016), S677-S681. 1, $2.1,2.2$

[24] X.-J. Yang, New integral transform method for solving steady heat-transfer problem, Therm. Sci., 20 (2016), S639-S642. 1

[25] X.-J. Yang, A new integral transform operator for solving the heat-diffusion problem, Appl. Math. Lett., 64 (2017), 193197. $1,2.2$

[26] X.-J. Yang, D. Baleanu, Y. Khan, S. T. Mohyud-Din, Local fractional variational iteration method for diffusion and wave equations on Cantor sets, Romanian J. Phys., 59 (2014), 36-48. 1

[27] X.-J. Yang, Y.-G. Yang, C. Cattani, M.-Z. Zhu, A new technique for solving the 1-D Burgers equation, Therm. Sci., 21 (2017), S129-S136. 1 